\title{
DAS PROPRIUM DES SPÄTMITTELALTERLICHEN UND FRÜHNEUZEITLICHEN HOSPITALS
}

\author{
Zusammenfassung
}

Was waren die Erwartungen? Die Tagung hatte sich ausdrücklich nicht das Ziel gesetzt, nach dem Gemeinsamen der einzelnen Hospitäler zu suchen, sondern zunächst einmal ihre Heterogenität wahrzunehmen und ihre institutionelle Vielfalt zu erforschen'. Das hieß fürs erste, sich auf eine Vielzahl von Themen einzulassen, die von der inneren Verfassung der Hospitäler, von den für sie respektive in ihnen geltenden Normen über die Finanzierung ihrer Wohltätigkeit und über ihr soziales Leben, wie es sich etwa in der memoria ausdrückt, bis hin zu ernährungsgeschichtlichen und medizinhistorischen Fragen reicht. Dabei leitete uns die Erkenntnis, daß es das mittelalterliche Hospital nicht gab, sondern daß jede einzelne Einrichtung, jeder Hospitalverbund viele unterschiedliche institutionelle Details in unterschiedlichem Ausmaße aufweist. Somit ist jedes konkrete Hospital vorerst einmal von einzigartiger Beschaffenheit, gleichsam ein Spiegel der Welt, die es umgibt. Diese Individualität galt es zu beachten, obwohl sie den Vergleich erheblich erschwert. Dennoch erschien eine Gegenüberstellung deutscher, französischer und italienischer Hospitäler als wünschenswert, nicht zuletzt, weil die Forschung, wie weiter oben einleitend dargestellt, in den genannten Ländern bereits seit langem in besonderem Maße aktiv ist. Das Kolloquium strebte also ganz bescheiden das Ziel an, die durch unterschiedliche methodische Ansätze und unterschiedliche Quellen gewonnenen Ergebnisse jüngerer Forscher einander gegenüberzustellen.

Was hat die Tagung erbracht? Es mag erstaunen, daß gerade an dieser Stelle als Quintessenz eines intensiven Arbeitstages der Versuch gewagt wird, das Proprium, das Eigene, das Wesenstypische des spätmittelalterlichen Hospitals vor allem in seiner Abgrenzung gegenüber anderen Einrichtungen zu skizzieren. Dieses Vorhaben erscheint mir aber als durchaus sinnvoll. Denn die Alternative wäre gewesen, die oben präsentierte Flut von Detailinformationen zu

${ }^{1}$ Gisela DrossBaCH, Hopitaux en France, en Allemagne et en Italie. Une histoire comparée (Moyen Âge et Temps Modernes) - Hospitäler in Frankreich, Deutschland und Italien. Eine vergleichende Geschichte (Mittelalter und Neuzeit), in: Zeitschrift der Savigny-Stiftung für Rechtsgeschichte 121, Kanonistische Abt. 90 (2004) S. 638-641. 
bündeln und zu kategorisieren. Doch wie sollte dies angesichts von unterschiedlichen Quellenbeständen, unterschiedlichen Strukturen, unterschiedlichen politischen, sozialen und wirtschaftlichen Wirklichkeiten geschehen, die zudem alle dem allgemeinen historischen Wandel, dem `Zeitgeistı des Spätmittelalters und der beginnenden Modeme, unterworfen waren? Außerdem ist die Auswahl der vorgestellten Objekte weder hinreichend noch zwingend noch repräsentativ, was meines Erachtens ein solches Unterfangen noch arbiträrer gemacht hätte. Ich bin mir durchaus bewußt, daß ich im Folgenden meinen ganz persönlichen Ertrag beschreibe - andere Teilnehmer haben gewiß anderes von der Tagung mit nach Hause genommen.

Ausgehend von der an sich banalen Feststellung, daß das Hospital eine Institution zwischen Erde und Himmel, zwischen Laien und Kirche oder, besser gesagt, Kloster war, dennoch beiden die mittelalterliche Welt so sehr prägenden Lebenskreisen in erheblichem, aber von Institution zu Institution variierendem Maße angehörte, verstehe ich das Hospital im Wesentlichen funktional.

Welche Elemente also gehören den beiden Lebenswelten an? Das Vorbild für den meist einschiffigen Hallenbau des spätmittelalterlichen Spitals stammt zweifellos aus der kirchlich-monastischen Welt. Der frühe städtische Spitalbau, der durchaus auch einen Kreuzgang und weitere Gebäude umfassen konnte, orientierte sich an den eher schmucklosen Bauten der frühen Bettelordenskirchen. Der Krankensaal gleicht architektonisch einer Kirche, worauf auch der an prominenter Stelle errichtete Altar hinweist, und nimmt - mindestens aus heutiger Sicht - zunächst auf seinen eigentlichen Verwendungszweck kaum Rücksicht. Ist der Krankensaal verziert, folgt sein Schmuck ebenfalls einem kirchlichen Vorbild, wobei der thematische Schwerpunkt des Bildprogramms interessanterweise häufig bei den sogenannt letzten Dingen liegt. Besonders beliebt scheinen Bilder des Jüngsten Gerichts gewesen zu sein, das unmittelbar an die Endlichkeit des menschlichen Daseins erinnert. Die erbauliche Ermahnung an die zeitliche Beschränktheit unserer Existenz auf Erden (oder unserer Anwesenheit im Spital) übte auf die Verantwortlichen offenbar eine besonders große Faszination aus. Während die memoria als Aufgabe eher dem monastischen Lebenskreis zuzuordnen ist, oblag die caritas zweifellos auch jedem Laien. Doch wesentlich ist, daß memoria und caritas das Hospital gemeinsam prägten, wie mehrfach gezeigt wurde. Zudem machte sich die ,Welt‘ im Laufe der Zeit im Hospital - früher und stärker als in anderen kirchlichen Institutionen - durch eine immer intensiver werdende politische und administrative Kontrolle bemerkbar.

Das Spital unterscheidet sich prinzipiell sowohl von monastischen wie auch von laikalen Institutionen darin, daß es im Gegensatz zu beiden gleichzeitig zwei Gemeinschaften mit je eigenen Interessen und Bedürfnissen in sich beherbergt, nämlich die Gemeinschaft des >Personals` und jene der IInsassen', die aber untrennbar miteinander verbunden, ja sogar grundsätzlich aufeinander 
angewiesen waren. Denn die eine konnte ohne die andere im Spital nicht existieren. Dieses Merkmal dürfte für alle Hospitäler in variablem Maße gegolten haben - mit Ausnahme vielleicht der Häuser für Sondersiechen, was sich aber medizinhistorisch erklären läßt. Die beiden Gruppen innerhalb des Spitals unterscheiden sich vor allem bezüglich der Dauer ihrer jeweiligen Anwesenheit in der Einrichtung. Das Personal gehörte vom Zeitpunkt des freiwilligen Beitritts an idealerweise unwidernuflich zum Hospital. Die Insassen hingegen, seien es nun Waisen, Verletzte, chronisch Kranke, Alte oder Pilger, verbrachten nur einen bestimmten, vom Zweck des Spitals definierten Zeitabschnitt ihres Lebens darin. Die Insassen gehörten dem Hospital also nur für einen begrenzten Zeitraum an, weshalb sich auch ihre Rechtsstellung von jener des Personals unterscheidet.

Das Personal des Spitals könnte man als das Subjekt der caritas bezeichnen. Es unterscheidet sich von den Laien, aber auch von den Insassen durch seine Tracht, deren schlichte Gestaltung die individuelle Armut ausdrückt und sich oft an den damals führenden religiösen Bewegungen orientierte. Die >corporate identity، des Personals, um dieses hier durchaus passende Modewort zu verwenden, drückte sich gelegentlich auch in einem Abzeichen aus, etwa dem Tau von Altopascio oder der Leiter von Santa Maria della Scala. Diese Zeichen wiederum schieden ihre Träger deutlich von der restlichen Welt und machten sie überall als Angehörige des Hospitals erkennbar. Weitere monastische Elemente finden sich im Gelübde auf Armut, Gehorsam und Keuschheit, die der traditionellen Trias für kirchliche Gemeinschaften entsprechen, sowie in den Geboten, täglich am Gottesdienst teilzunehmen und regelmäßig zu beichten. Schließlich stammen auch der gemeinsame EB- und Schlafsaal aus der klösterlichen Kultur. Das Gehorsamkeitsgelübde hatte bei Verstößen und Ungehorsam Strafen zur Folge. Das Spital unterschied sich jedoch vom Kloster, weil es keine absolute Klausur kannte, sondern seinem Personal tagsüber Freigang erlaubte und es meistens nur in der Nacht einschloß. Wer immer wieder hartnäckig gegen die Regeln verstieß, dem drohte im Extremfall der Ausschluß aus der Gemeinschaft. Er wurde aber nicht eingekerkert, wie wir es bei ungehorsamen Mönchen und Nonnen kennen. $\mathrm{Zu}$ guter Letzt manifestierte sich der Gottesdienst im Hospital als Hinwendung zum Mitmenschen und nicht als Weltflucht in Gebet und Andacht. In den Nutznießern des Spitals erkannte man Christus. Dem Herm diente man, indem man den Bedürftigen half. Die Regeln und Statuten des Spitals sowie das Gelöbnis ließen für das Personal einen gegenüber der Laienwelt privilegierten Raum entstehen, was sich möglicherweise motivierend auf den Beitritt zur Gemeinschaft ausgewirkt hatte. Die tägliche Arbeit bestand fortan im Dienst am anderen. Der Verzicht auf persönliches Gewinnstreben fand im »ewigen Lohn « seinen Ersatz.

Die Insassen des Hospitals ihrerseits waren die Objekte der caritas. Ganz allgemein gesprochen bildeten sozial oder ökonomisch Benachteiligte oder 
Bedürftige die Zielgruppe des Hospitals. Ihnen kamen die Werke der Barmherzigkeit zugute, welche das Spitalpersonal täglich erbrachte. Daher funktionierte das Hospital genauso gut als Haus für Findelkinder wie für Leprosen, als Herberge für Heimatlose und Reisende wie als Pflegeanstalt für Verletzte, chronisch Kranke oder Alte. Natürlich waren auch die Insassen gewissen Vorschriften unterworfen, doch war die sie betreffende Regelungsdichte weit geringer. Meistens waren sie auch weniger privilegiert als das Personal.

Auch wenn der Hauptzweck des Hospitals die gelebte caritas war, so erschöpfte er sich nicht darin. Die beiden Gemeinschaften des Personals und der Insassen bildeten Tag für Tag nicht nur eine Gebets- und eine Gedächtnisvereinigung, sondern eben auch eine Gemeinschaft von Lebenden und Toten, weshalb oft ein Friedhof zum Hospital gehörte.

Schließlich war das Hospital ein Hort der medizinischen Versorgung, zunächst einmal auf der Basis der Volksheilkunde, später aber immer stärker auch unter Einbeziehung der akademischen Medizin. Die dem Hospital obliegende cura corporis et animae verbindet daher formelhaft die beiden Pole Welt und Kirche, zwischen denen sich das Hospital befand: in der Welt wurzelten Schutz, Ernährung und medizinische Pflege (cura corporis), in der Kirche die Seelsorge (cura animae).

Was wäre noch wünschenswert? Ohne $Z$ weifel die weitere Erschließung und Auswertung von bislang unberücksichtigten Quellen auf der Ebene der einzelnen Hospitäler. Dabei sollten aber die Quellen nicht vergessen werden, die wie etwa Ratsprotokolle oder Notarsregister Hospitäler nur indirekt betreffen, denn auch sie sind im Einzelfall äußerst fruchtbar.

Auf der Ebene der mehr hospitalbezogenen Quellen drängt sich bei den Ordnungen oder Statuten eine vergleichende Analyse auf, wobei man meines Erachtens dabei >Einhaushospitäler` und >Mehrhaushospitäler gesondert betrachten müßte. Die Statuten der ordensähnlichen Hospitalverbünde (Santo Spirito in Sassia, Altopascio, Antoniter und ähnliche) wären außerdem hinsichtlich ihres Vorbildes und ihrer gegenseitigen Abhängigkeit genauer zu untersuchen. Einhaushospitäler unterscheiden sich wiederum bezüglich ihrer Stifter und des Zeitpunktes ihrer Stiftung so sehr, daß möglicherweise das Gemeinsame im Unterschiedlichen untergeht. Zudem sollte dabei die Problematik des mittelalterlichen Stiftungsrechtes nicht vergessen werden. Weiter ist daran zu denken, daß in den Hospitälern die Regelungsdichte mit der Dauer der Existenz der Einrichtung enorm ansteigt.

Brauchen wir eine Bestandsaufnahme aller abendländischen Hospitäler mit anschließender Auswertung hinsichtlich Stifter, Entstehungszeit, Finanzierung und Funktion? - Für einen abendländischen Hospitälerkatalog, der knapp und präzise über die Geschichte der einzelnen Institutionen informiert, könnte ich mich durchaus begeistern. Denn als Forscher begegnet man den Hospitälern wegen ihren vielfältigen Funktionen in vielen Zusammenhängen und oft wüß- 
te man gerne mehr über diese Einrichtungen. Dennoch erscheinen mir persönlich die individuellen Züge der einzelnen Institutionen als zu gewichtig, als zu prägend, als daß sich das Fernziel einer »Histoire totale des hôpitaux « unmittelbar aufdrängt. 
